\title{
Frontières
}

\section{Le vieillissement chez les hommes gais et bisexuels canadiens : un portrait de l'état de santé et des préoccupations relatives à la santé et aux relations interpersonnelles}

\author{
Joseph J. Lévy, Barry Adam, Martin Blais, Line Chamberland, Jean Dumas, Kim \\ Engler, Alain Léobon, Bill Ryan, Christine Thoër et Kristopher Wells
}

Volume 25, numéro 1, automne 2012

Le vieillissement et sa diversité

URI : https://id.erudit.org/iderudit/1018232ar

DOI : https://doi.org/10.7202/1018232ar

Aller au sommaire du numéro

Éditeur(s)

Université du Québec à Montréal

ISSN

1916-0976 (numérique)

Découvrir la revue

Citer cet article

Lévy, J. J., Adam, B., Blais, M., Chamberland, L., Dumas, J., Engler, K., Léobon, A. Ryan, B., Thoër, C. \& Wells, K. (2012). Le vieillissement chez les hommes gais et bisexuels canadiens : un portrait de l'état de santé et des préoccupations relatives à la santé et aux relations interpersonnelles. Frontières, 25(1), 82-104. https://doi.org/10.7202/1018232ar
Résumé de l'article

Peu de recherches ont porté sur les profils et les préoccupations de santé parmi les hommes homosexuels et bisexuels vieillissants. Dans le cadre d'une enquête en ligne pancanadienne, 411 répondants âgés de 55 ans et plus ont répondu à un questionnaire portant sur l'évaluation de ces deux problématiques. Les résultats montrent que les écarts avec la population hétérosexuelle du même groupe d'âge se situent en particulier dans le champ de la santé mentale, où les problèmes sont plus prononcés, des différences qui se retrouvent aussi entre les 55-64 ans et les 64 ans et plus dans notre échantillon. Ces résultats peuvent contribuer à développer des interventions mieux ciblées visant à favoriser le bien-vieillir parmi ces minorités sexuelles.
Tous droits réservés (C Université du Québec à Montréal, 2013

Ce document est protégé par la loi sur le droit d'auteur. L'utilisation des services d'Érudit (y compris la reproduction) est assujettie à sa politique d'utilisation que vous pouvez consulter en ligne.

https://apropos.erudit.org/fr/usagers/politique-dutilisation/ 


\section{LE VIEILLISSEMENT CHEZ LES HOMMES GAIS ET BISEXUELS CANADIENS : UN PORTRAIT DE L'ÉTAT DE SANTÉ ET DES PRÉOCCUPATIONS RELATIVES À LA SANTÉ ET AUX RELATIONS INTERPERSONNELLES ${ }^{1}$}

Joseph J. Lévy, Ph.D.

Département de sexologie, Université du Québec à Montréal

Barry Adam, Ph.D. Department of sociology, anthropology and criminology, University of Windsor

Martin Blais, Ph.D. Département de sexologie, Université du Québec à Montréal

Line Chamberland, Ph.D. Département de sexologie, Université du Québec à Montréal

Jean Dumas, Ph.D. Département de sexologie, Université du Québec à Montréal

Kim Engler, Ph.D. Département de sexologie, Université du Québec à Montréal

Alain Léobon, Ph.D.

CNRS France

Bill Ryan, M.S.W. School of Social Work, McGill University

Christine Thoër, Ph.D. Département de communications, Université du Québec à Montréal

Kristopher Wells, Ph.D. Department of educational policy studies, University of Alberta 
Peu de recherches ont porté sur les profils et les préoccupations de santé parmi les hommes homosexuels et bisexuels vieillissants. Dans le cadre d'une enquête en ligne pancanadienne, 411 répondants âgés de 55 ans et plus ont répondu à un questionnaire portant sur l'évaluation de ces deux problématiques. Les résultats montrent que les écarts avec la population hétérosexuelle du même groupe d'âge se situent en particulier dans le champ de la santé mentale, où les problèmes sont plus prononcés, des différences qui se retrouvent aussi entre les 55-64 ans et les 64 ans et plus dans notre échantillon. Ces résultats peuvent contribuer à développer des interventions mieux ciblées visant à favoriser le bien-vieillir parmi ces minorités sexuelles.

\section{ABSTRACT}

Few studies have analyzed health profiles and concerns profiles among aging homosexual and bisexual men. Using a subsample from a pan-Canadian online survey, data on self-perceived health problems of 411 participants aged 55 years and more have been analyzed. Results show that the differences with the heterosexual population of the same age categories are present, especially in the sphere of mental health, where problems are more acute. Some differences are also present between the 55-64 and 65 and more age groups in our sub-sample. These results can help the development of better interventions in order to contribute to the well-aging among these sexual minorities.

MOTS CLÉS: homosexuels - bisexuels - vieillissement - bien-être santé - Canada

KEYWORDS: homosexuals - bisexuals - aging - well - being - health - Canada 
Plusieurs chercheurs se sont attachés à dégager chez les minorités sexuelles les profils et les préoccupations touchant leur santé physique et mentale de même que leur qualité de vie (Meyer et Northridge, 2007). Aux États-Unis, des études comparatives ont montré que les hommes d'orientation gaie et bisexuelle sont confrontés à des problèmes de santé plus fréquents que les hommes hétérosexuels (consommation de drogues, tentatives de suicide et idéations suicidaires, troubles de santé mentale; Haas, Eliason, Mays et al., 2011; King, Semlyen, Tai et al., 2008). Ces problèmes trouveraient notamment leur source dans le contexte social défavorable à l'égard des LGBT (homophobie, marginalisation et stress liés au statut minoritaire; Meyer, 2007) et ils interagiraient et se renforceraient mutuellement (Stall, Friedman et Catania, 2008).

Au Canada, même si, de façon générale, le contexte social semble un peu plus favorable aux minorités sexuelles (Smith, 2005; Tjepkema, 2008) et les services de santé plus accessibles, les problèmes de santé restent importants. Les hommes gais cumuleraient ainsi plus de problèmes de santé chroniques diagnostiqués par un professionnel de la santé (de plus de six mois; Tjepkema, 2008), mais tant les hommes gais que bisexuels auraient plus tendance que les hétérosexuels à recourir à des services de soutien émotionnel et psychologique. Une étude basée sur un échantillon national collecté en 2003 (Brennan, Ross, Dobinson, Veldhuizen et Steele, 2010) a cerné des profils de santé dans cette population. Contrairement aux résultats d'autres recherches, les hommes gais et bisexuels ne sont pas plus nombreux à rapporter des affections respiratoires ou des niveaux de consommation plus élevés d'alcool ou de cigarettes que les hétérosexuels. Ils sont moins enclins à souffrir de surpoids ou d'obésité. Ils ne diffèrent pas non plus quant à l'évaluation subjective de leur santé physique ou mentale. Par contre, la prévalence à vie des infections transmissibles sexuellement (ITS), des troubles de I'humeur, des troubles anxieux et des tendances suicidaires est significativement plus élevée.

D'autres recherches se sont attachées à considérer les problématiques de santé parmi les minorités sexuelles en fonction des groupes d'âge, comme les jeunes (Saewyc et al., 2009; Dysart-Gale, 2010) et les hommes gais et bisexuels plus âgés (40 ans et plus), mettant en évidence des variations dans l'état de santé physique et mentale et l'importance pour le bien vieillir (successful aging), de facteurs comme 
la cohorte de naissance, l'origine ethnique, la classe sociale, le niveau de support et l'implication communautaire (Mayock et al., 2009; Fredriksen-Goldsen et Muraco, 2010; Grant, 2010; Higgins et al., 2011; Committee on Lesbian, Gay, Bisexual, and Transgender Health Issues and Research Gaps and Opportunities Board on the Health of Select Populations, 2011). Les facteurs liés à l'acceptation et à la gestion de l'identité gaie ou lesbienne et l'implication dans une relation de couple contribuent à une bonne santé mentale alors que l'isolement, une faible estime de soi, une homophobie intériorisée et une victimisation liée à l'orientation sexuelle sont des déterminants d'une santé mentale problématique, marquée par des idéations suicidaires, des tentatives de suicide et I'usage de drogues (Fredriksen-Goldsen et Muraco, 2010).

La discrimination peut en particulier intervenir dans I'utilisation et l'accès aux services de santé et limiter leur recours, souvent moins fréquent que parmi les hétérosexuels (Fredriksen-Goldsen et Muraco, 2010) et cette situation s'observe au Canada. Selon l'étude qualitative de Brotman, Ryan et Cormier (2003) les ainés, gais et lesbiennes, du Québec, de la Nouvelle-Écosse et de la Colombie-Britannique sont confrontés à de nombreux obstacles dans l'accès aux services de santé (discrimination, soins inadaptés aux orientations sexuelles). À Vancouver, selon des entrevues auprès de personnes âgées, dans leur majorité de 65 ans et plus et provenant de ces mêmes minorités (Johnston, 2008), la grande majorité d'entre eux disait avoir une santé excellente ou bonne et faire preuve d'autonomie dans les activités quotidiennes. Dans l'évaluation de la santé physique, aucun des répondants ne rapportait être infecté par le VIH, mais plusieurs souffraient ou avaient souffert d'un cancer. Leurs préoccupations majeures portaient sur l'exercice, l'alimentation ainsi que la gestion de la médication et des thérapies alternatives. Plusieurs diagnostics de troubles de santé mentale au cours de leur vie étaient mentionnés, en particulier la dépression, qui continuait à affecter près de la moitié des répondants, et l'anxiété, mais très peu d'entre eux consommaient des drogues. Dans leur rapport aux services de santé, la majorité des répondants avait discuté de leur orientation sexuelle avec leur médecin, mais les lesbiennes étaient plus nombreuses que les hommes gais à rapporter avoir été confrontées à des attitudes homophobes ou hétérosexistes de la part des intervenants en santé. Plusieurs estimaient aussi avoir des besoins spécifiques en termes de soins qui n'étaient pas comblés par les 
services actuels. Pour les différentes sphères de vie, la majorité des personnes interrogées se disaient satisfaites, que ce soit par rapport à leur sexualité, qu'ils soient ou non en relation avec un/e partenaire; à leur réseau social (famille et amis) dont le soutien qu'ils en recevaient était jugé adéquat; ou de leur existence en général, malgré une vision souvent négative du vieillissement, notamment en raison des changements qui affectaient leur image corporelle.

Ces recherches qualitatives permettent de cerner certains des profils de santé présents dans ces minorités sexuelles vieillissantes sans pouvoir cependant vérifier leur prévalence ou dégager plus largement le registre des préoccupations dans ce domaine. Nous tenterons de compléter ces analyses en présentant les résultats d'une recherche quantitative menée auprès d'un échantillon d'hommes homosexuels et bisexuels âgés de 55 ans et plus, recrutés en ligne. Cette évaluation des profils et des préoccupations, qui sera comparée dans la mesure du possible avec ceux de la population générale, aidera à mieux cerner les problèmes de santé rencontrés et contribuera à mieux adapter les programmes de prévention et de promotion de la santé pour ces populations en tenant compte de leurs besoins spécifiques.

\section{MÉTHODOLOGIE}

\section{- Procédures}

Cette enquête pancanadienne en ligne a été conduite auprès des personnes lesbiennes, gaies, bisexuelles, bispirituelles ${ }^{2}$ et transgenres avec comme objectif de recueillir des informations sur leur état santé, leurs préoccupations de santé et les usages qu'ils font d'Internet à des fins de santé. Afin de publiciser cette enquête, des organismes LGBTQ et de lutte contre le VIH/sida ont accepté de distribuer du matériel informationnel sur la recherche. Une bannière a été affichée en ligne durant 2 mois sur un site web d'actualités pour les gais et lesbiennes du Canada et une annonce a été placée dans le bulletin électronique distribué à ses membres. Une invitation à participer à l'étude a été envoyée par courriel personnalisé aux membres de deux sites de rencontres pour hommes gais ainsi qu'aux membres de groupes LGBT en ligne basés au Canada. La participation à l'étude, qui avait reçu 
l'approbation du Comité institutionnel d'éthique de la recherche avec des êtres humains de I'Université du Québec à Montréal, était volontaire et ne comportait aucune compensation.

Le recrutement s'est déroulé de juillet à décembre 2010 et 2187 questionnaires ont été remplis. Plus des trois quarts (76.1\%) des participants sont des hommes ayant des relations sexuelles avec d'autres hommes, exclusivement ( $n=1110 ; 50,8 \%$ ) ou non ( $n=564$; $25,3 \%$ ). De ce nombre, seuls les répondants de 55 ans et plus ont été inclus dans l'analyse $(n=411)$. Ils ont été répartis en deux groupes d'âge: 55-64 ans $(n=311)$ et 65 ans et plus $(n=100)$, cette catégorisation rejoignant celle généralement employée dans les études sociodémographiques.

\section{Variables}

\section{Variables sociodémographiques}

Les variables suivantes ont été incluses dans les analyses à titre de variables de contrôle: la langue du questionnaire (français ou anglais), l'âge (en année), l'orientation sexuelle (gai ou bisexuel, définie en fonction du sexe des partenaires), le statut de travailleur (temps plein ou partiel), le statut de retraité (oui ou non), la scolarité (plus qu'un diplôme d'études secondaires ou non), le statut relationnel (en couple ou non), le statut migratoire (immigrant de 1ère génération, de $2^{\mathrm{e}}$ génération ou de $3^{e}$ génération ou plus), le revenu annuel brut (30 000 dollars ou plus, moins de 30000 ), la pratique religieuse (échelle de 1 , jamais ou pratiquement jamais, à 8 , plus d'une fois par semaine), et le statut sérologique pour le VIH (séropositif ou séronégatif).

\section{Indicateurs de l'état de santé}

L'état de santé perçu (évaluation subjective) a été évalué par deux énoncés sur la santé générale et la santé mentale, dont l'échelle de réponse en 5 points variait d'excellente à mauvaise. Les réponses ont été dichotomisées en deux catégories: (0) mauvaise à bonne et (1) très bonne à excellente. Quatre énoncés ont permis de mesurer la présence (oui ou non) de diagnostics (1) de maladie chronique, (2) de santé physique autre que chronique, (3) de santé mentale et (4) de 
santé sexuelle. Le fait d'avoir ou non un médecin de famille attitré ainsi qu'un diagnostic de séropositivité au VIH ont aussi été questionnés.

\section{Préoccupations relatives à la santé et aux relations interpersonnelles}

Les répondants devaient indiquer s'ils avaient été, au cours des 12 mois précédant l'enquête, personnellement confrontés à 35 préoccupations relatives à la santé et aux relations interpersonnelles. Les préoccupations étaient décrites succinctement aux répondants et couvraient les cinq dimensions suivantes: la santé physique, la santé mentale, la santé sexuelle, les habitudes de consommation de substances et les relations interpersonnelles (le Tableau 3 ci-après présente le détail des préoccupations incluses dans chaque catégorie).

\section{Analyses}

Dans un premier temps, les deux groupes d'âge ont été comparés sur l'occurrence des préoccupations de santé à l'aide de Chi-carrés de Pearson (pour mesurer l'association entre deux variables). Lorsque les effectifs dans une catégorie étaient inférieurs à 5 , le test exact de Fisher a été utilisé pour les variables catégorielles. Dans un second temps, des analyses de régression (afin de dégager les variables les plus déterminantes) ont été effectuées avec, pour variable dépendante, celles sur lesquelles les groupes se distinguaient. La variable de groupe d'âge a été utilisée comme variable indépendante et les variables suivantes ont été incluses à titre de variables de contrôle: la langue, le revenu, la scolarité, la pratique religieuse, l'orientation sexuelle, le statut migratoire, le statut sérologique et le statut relationnel. Étant donné que les groupes ne se sont distingués que sur des variables dichotomiques, des modèles logistiques ont été estimés. Seuls les ratios de cote de la variable indépendante pour les modèles qui présentent des différences significatives sont rapportés. La taille des effectifs varie en fonction des variables étudiées en raison du patron monotone de données manquantes attribuable à l'abandon des répondants au cours de la recherche. 


\section{RÉSULTATS}

Le Tableau 1 présente les caractéristiques sociodémographiques des répondants. Dans le groupe des 55-64 ans, on retrouve des proportions plus élevées de répondants ayant rempli le questionnaire en français ( $46 \%$ c. $34,3 \%$ chez les 65 ans et plus) et occupant un emploi à temps plein ( $50,0 \%$ c. $22,4 \%$ chez les 65 ans et plus). Dans le groupe des 65 ans ou plus, on trouve des proportions plus élevées de retraités (65,4\% c. $29,4 \%$ chez les 55-64 ans) et d'immigrants de $2^{\mathrm{e}}$ génération ( $17,3 \%$ c. 8,1 chez les 55-64 ans). Les 65 ans et plus sont aussi marginalement plus nombreux à détenir un niveau de scolarité supérieur au DES (84,3\% c. $75,3 \%$ chez les $55-64$ ans), mais rapportent aussi marginalement en moins grand nombre gagner un revenu annuel supérieur à 30000 \$ (69,2\% c. 78,6\% chez les 55-64 ans). Les groupes ne se distinguent pas significativement quant aux proportions de répondants en couple et d'orientation bisexuelle.

\section{Tableau 1 Caractéristiques sociodémographiques des participants}

\begin{tabular}{|c|c|c|c|c|c|}
\hline & \multirow[b]{2}{*}{$\begin{array}{l}\text { Total } \\
(\%)\end{array}$} & \multicolumn{2}{|c|}{ Groupes d'âge } & \multirow[b]{2}{*}{$X^{2(d l=1)}$} & \multirow[b]{2}{*}{$p$} \\
\hline & & $\begin{array}{c}55-64 \\
(\%)\end{array}$ & $\begin{array}{l}65 \text { et } \\
\text { plus } \\
(\%)\end{array}$ & & \\
\hline $\begin{array}{l}\text { Langue du questionnaire } \\
\text { (français) }\end{array}$ & 43,0 & 46,0 & 34,3 & 4,520 & 0,033 \\
\hline Emploi (temps plein) & 42,9 & 50,0 & 22,4 & 24,68 & 0,000 \\
\hline Retraité & 38,6 & 29,4 & 65,4 & 43,65 & 0,000 \\
\hline \multicolumn{6}{|l|}{ Immigrant } \\
\hline $1^{\text {re }}$ génération & 12,8 & 12,6 & 13,5 & 7,49 & 0,024 \\
\hline $2^{\mathrm{e}}$ génération & 10,4 & 8,1 & 17,3 & & \\
\hline 3e génération ou plus & 76,8 & 79,4 & 69,2 & & \\
\hline Scolarité (plus que DES) & 77,5 & 75,3 & 84,3 & 3,60 & 0,058 \\
\hline Revenu (plus que 30 000) & 76,2 & 78,6 & 69,2 & 3,77 & 0,052 \\
\hline Statut relationnel (couple) & 45,4 & 45,3 & 45,6 & 0,003 & 0,954 \\
\hline $\begin{array}{l}\text { Orientation sexuelle } \\
\text { (bisexuel) }\end{array}$ & 36,4 & 34,4 & 42,1 & 2,01 & 0,156 \\
\hline
\end{tabular}




\section{Profils de santé des répondants}

Parmi l'ensemble des répondants, 58,1\% considéraient leur état de santé en général comme excellent ou très bon, sans distinction significativement entre les deux groupes. Près des trois quarts $(73,4 \%)$ d'entre eux se considéraient en excellente ou en très bonne santé mentale, surtout chez les répondants de 65 ans et plus, comparativement à ceux de 55-64 ans. Cette différence se maintient lorsque I'on tient compte des caractéristiques sociodémographiques (voir Tableau 4 pour les ratios de cote ajustés en fonction des variables de contrôle).

Pour l'ensemble des répondants, 49,7\% rapportaient au moins une maladie chronique; $40,0 \%$ au moins un problème de santé physique; $15,1 \%$ au moins un problème de santé mentale et $19,3 \%$ au moins un problème de santé sexuelle. Les deux groupes d'âge ne se distinguent significativement que sur le plan de la santé mentale, les 65 ans et plus étant moins nombreux (9,2\% c. $17,5 \%$ des 55-64 ans) à avoir vécu des difficultés à cet égard au cours de l'année ayant précédé l'enquête. Cette différence se maintient même après ajustement pour les variables de contrôle (Tableau 4).

Un peu plus du dixième des hommes interrogés ont rapporté être infectés par le VIH. Cette proportion était significativement plus élevée dans le groupe des $55-64$ ans (13,5\% c. 5,1\% chez les 65 ans et plus), même une fois ajustée pour les variables de contrôle (Tableau 4). Une très grande majorité $(88,5 \%)$ des répondants confirmaient avoir un médecin de famille et cette proportion était marginalement plus élevée chez les 65 ans et plus (93,9\% c. 86,7\% chez les 55-64 ans).

\section{Tableau 2 Profil de santé des répondants}

\begin{tabular}{|c|c|c|c|c|c|}
\hline & \multirow[b]{2}{*}{ Total } & \multicolumn{2}{|c|}{ Groupes d'âge } & \multirow[b]{2}{*}{ Statistiques } & \multirow[b]{2}{*}{$p$} \\
\hline & & $55-64$ & $\begin{array}{l}65 \text { et } \\
\text { plus }\end{array}$ & & \\
\hline $\begin{array}{l}\text { Variables } \\
\text { dichotomiques (\%) }\end{array}$ & & & & $X^{2(d l=1)}$ & \\
\hline \multicolumn{6}{|l|}{$\begin{array}{l}\text { Auto-évaluation de l'état } \\
\text { de santé (excellente } \\
\text { ou très bonne) }\end{array}$} \\
\hline santé générale & 58,1 & 59,3 & 54,6 & 0,695 & 0,405 \\
\hline santé mentale & 73,4 & 70,2 & 82,8 & 6,077 & 0,014 \\
\hline
\end{tabular}




$\begin{array}{lccccc}\begin{array}{l}\text { Conditions médicales } \\ \text { relatives à la } \\ \text { maladie chronique }\end{array} & 49,7 & 49,7 & 50,0 & 0,003 & 0,953 \\ \text { santé physique } & 40,0 & 39,0 & 42,9 & 0,445 & 0,505 \\ \text { santé mentale } & 15,4 & 17,5 & 9,2 & 3,866 & 0,049 \\ \text { santé sexuelle } & 19,3 & 21,2 & 13,3 & 2,999 & 0,083 \\ \text { Avoir un médecin de famille } & 88,5 & 86,7 & 93,9 & 3,73 & 0,054 \\ \text { Personnes vivant avec le VIH } & 11,4 & 13,5 & 5,1 & 5,22 & 0,022\end{array}$

\section{- Les préoccupations relatives à la santé et aux relations interpersonnelles}

Le Tableau 3 décrit la proportion de répondants ayant été personnellement confrontés à des préoccupations relatives à la santé et aux relations interpersonnelles. On constate une variation importante dans les proportions de répondants qui rapportent l'une ou l'autre des préoccupations. La préoccupation la plus fréquente concerne l'image corporelle $(38,8 \%)$ et la moins fréquente, la consommation des drogues $(2,0 \%)$. Dans le domaine de la santé physique, c'est le dépistage du cancer $(20,5 \%)$, suivi des problèmes cardiaques $(16,4 \%)$, qui dominent, une minorité faisant état de préoccupations concernant le traitement (5,5\%). Aucune différence entre les deux groupes d'âge n'est présente.

Dans le domaine de la santé mentale, la préoccupation relative à l'image corporelle est la plus répandue $(38,8 \%)$, suivie des préoccupations relatives à l'anxiété $(35,4 \%)$, à la solitude $(27,9 \%)$ et à la dépression $(26,1 \%)$. Les deux groupes d'âge diffèrent quant à la proportion de répondants préoccupés par l'image corporelle, la solitude et la dépression, toutes plus fréquentes chez les 55-64 ans. Après ajustement pour les variables de contrôle (Tableau 4), les groupes ne se distinguent plus que sur leur préoccupation pour la dépression (ratio de cote $(R C)=0,38$; intervalle de confiance (IC) $95 \%=0,18-0,80 ; p=0,011$ ), alors que I'image corporelle et la solitude deviennent marginalement significatives $(p<0,10)$.

Le cinquième des répondants rapporte avoir été confronté à la dépendance aux images érotiques et à Internet de même qu'à 
I'acceptation de son orientation sexuelle, alors que les autres préoccupations (idéations ou tentatives suicidaires, troubles de l'alimentation, acceptation de l'identité de genre, dépendance au jeu) rejoignent moins de $10 \%$ des répondants. Les deux groupes ne diffèrent a priori que sur la préoccupation à l'égard des troubles de I'alimentation, rapportée en plus grand nombre par les 55-64 ans, mais cette différence ne se maintient pas lorsque I'on tient compte des facteurs sociodémographiques (Tableau 4).

\section{Tableau 3 Préoccupations relatives à la santé et aux relations interpersonnelles auxquelles les répondants ont été confrontés au cours des 12 mois précédant l'enquête}

\begin{tabular}{|c|c|c|c|c|c|}
\hline \multirow[b]{2}{*}{ Préoccupation \% } & \multirow[b]{2}{*}{ Total } & \multicolumn{2}{|c|}{ Groupe d'âge } & \multirow[b]{2}{*}{$X^{2(d l=1)}$} & \multirow[b]{2}{*}{$p$} \\
\hline & & $55-64$ & $\begin{array}{l}65 \text { et } \\
\text { plus }\end{array}$ & & \\
\hline \multicolumn{6}{|l|}{ Santé physique } \\
\hline Dépistage du cancer & 20,5 & 21,4 & 17,6 & 0,516 & 0,473 \\
\hline Problèmes cardiaques & 16,4 & 14,5 & 22,1 & 2,694 & 0,101 \\
\hline Traitement du cancer & 5,5 & 4,7 & 8,3 & 1,406 & 0,236 \\
\hline \multicolumn{6}{|l|}{ Santé mentale } \\
\hline Image corporelle & 38,8 & 42,3 & 27,9 & 5,692 & 0,017 \\
\hline Anxiété & 35,4 & 37,6 & 28,4 & 2,265 & 0,132 \\
\hline Solitude ou isolement & 27,9 & 31,7 & 16,3 & 7,670 & 0,006 \\
\hline Dépression & 26,1 & 29,3 & 15,7 & 6,125 & 0,013 \\
\hline $\begin{array}{l}\text { Dépendance aux images } \\
\text { érotiques }\end{array}$ & 20,9 & 21,3 & 19,8 & 0,089 & 0,766 \\
\hline Dépendance à Internet & 20,6 & 20,9 & 19,8 & 0,049 & 0,824 \\
\hline $\begin{array}{l}\text { Acceptation de son } \\
\text { orientation sexuelle }\end{array}$ & 19,4 & 19,5 & 18,8 & 0,021 & 0,886 \\
\hline $\begin{array}{l}\text { Idéation ou tentative } \\
\text { suicidaire }\end{array}$ & 9,3 & 9,6 & 8,4 & 0,104 & 0,747 \\
\hline $\begin{array}{l}\text { Acceptation de l'identité } \\
\text { de genre }\end{array}$ & 8,9 & 8,4 & 10,5 & 0,335 & 0,563 \\
\hline Troubles de I'alimentation & 8,0 & 9,7 & 2,3 & 4,979 & $0,023+$ \\
\hline Dépendance au jeu & 2,8 & 3,3 & 1,2 & 1.092 & $0,462+$ \\
\hline
\end{tabular}




\section{Santé sexuelle}

Dépistage du VIH

$28,7 \quad 29,0 \quad 27,7 \quad 0,055 \quad 0,814$

Dépistage d'autres ITSS

$\begin{array}{lllll}27,8 & 28,7 & 24,7 & 0,523 & 0,469\end{array}$

Comportements sexuels

à risque

$26,4 \quad 28,2 \quad 20,0 \quad 2,145 \quad 0,143$

Prévention du VIH

$19,4 \quad 19,7 \quad 18,3 \quad 0,081 \quad 0,776$

Sexualité compulsive

$17,1 \quad 19$,

$9,3 \quad 4,846$

0,028

Traitement des ITSS

7,7

8,6

4,8

1,321

0,250

\section{Habitudes de}

\section{consommation}

\begin{tabular}{|c|c|c|c|c|c|}
\hline Tabagisme & 23,3 & 25,0 & 18,3 & 1,530 & 0,216 \\
\hline Consommation d'alcool & 13,5 & 14,1 & 11,8 & 0,305 & 0,581 \\
\hline Consommation de drogues & 2,0 & 1,9 & 2,4 & 0,082 & $0,675+$ \\
\hline \multicolumn{6}{|l|}{$\begin{array}{l}\text { Relations } \\
\text { interpersonnelles }\end{array}$} \\
\hline $\begin{array}{l}\text { Recherche de partenaire } \\
\text { stable }\end{array}$ & 28,6 & 29,9 & 24,7 & 0,836 & 0,360 \\
\hline Recherche d'amis & 25,1 & 27,1 & 18,6 & 2,526 & 0,112 \\
\hline Relation de couple actuel & 20,8 & 22,9 & 14,1 & 3,039 & 0,081 \\
\hline $\begin{array}{l}\text { Craintes que I'on découvre } \\
\text { son orientation sexuelle }\end{array}$ & 20,8 & 21,4 & 18,8 & 0,265 & 0,606 \\
\hline Relations familiales & 19,5 & 21,3 & 13,9 & 2,064 & 0,151 \\
\hline $\begin{array}{l}\text { Discrimination ou attitudes } \\
\text { négatives envers } \\
\text { I'orientation sexuelle }\end{array}$ & 17,9 & 19,6 & 12,2 & 2,354 & 0,125 \\
\hline Rupture d'une relation & 12,5 & 15,0 & 4,9 & 5,809 & $0,020+$ \\
\hline $\begin{array}{l}\text { Dévoilement de I'orientation } \\
\text { sexuelle (coming out) }\end{array}$ & 8,6 & 9,6 & 5,8 & 1,216 & 0,270 \\
\hline $\begin{array}{l}\text { Discrimination en raison } \\
\text { de la séropositivité }\end{array}$ & 7,5 & 8,7 & 3,6 & 2,414 & $0,154+$ \\
\hline Violence en général & 4,8 & 5,9 & 1,2 & 3,125 & $0,086+$ \\
\hline $\begin{array}{l}\text { Violence dans la relation } \\
\text { de couple }\end{array}$ & 3,1 & 4,0 & 0,0 & 3,519 & $0,073+$ \\
\hline $\begin{array}{l}\text { Discrimination ou attitudes } \\
\text { négatives envers l'identité } \\
\text { de genre }\end{array}$ & 3,2 & 3,0 & 3,5 & 0,0500 & $0,734+$ \\
\hline
\end{tabular}

† Valeur $p$ obtenue par le test exact de Fisher. 
Dans le domaine de la santé sexuelle, plus du quart des répondants se disent préoccupés par le dépistage du VIH/sida et des autres ITSS ainsi que par les comportements sexuels à risque, alors que près de $20 \%$ des répondants le sont par la prévention du VIH. Aucune différence entre les groupes n'est observée sur ces préoccupations. La sexualité compulsive interpelle $17 \%$ des répondants et les 55-64 ans sont significativement plus enclins à se dire préoccupés par celle-ci, une différence qui ne devient que marginalement significative lorsque l'on tient compte des facteurs sociodémographiques ( $R C=0,39 ;$ IC95\% $=$ $0,15-1,02 ; p=0,054)$. Quant à la préoccupation pour le traitement des ITSS, moins de $10 \%$ des répondants en font mention et les groupes ne se distinguent pas à cet égard.

Parmi les habitudes de consommation, le tabagisme retient l'attention de près de $25 \%$ des répondants, suivi de la consommation d'alcool rapportée par 13,5 d'entre eux, mais la préoccupation relative à la consommation de drogues n'est rapportée que par $2 \%$ des répondants. Les deux groupes ne se distinguent pas en regard de cet ensemble de préoccupations.

Quant aux relations interpersonnelles, la quête d'un partenaire stable constitue la préoccupation la plus rapportée, et ce, par près du tiers des répondants, suivie par celle touchant la recherche d'amis pour le quart des participants. Les préoccupations relatives à la relation de couple actuelle, à la découverte de leur orientation actuelle et aux relations familiales sont aussi rapportées par environ le cinquième des participants. La confrontation aux enjeux relatifs à la discrimination ou aux attitudes négatives envers l'orientation sexuelle est rapportée par environ $18 \%$ des répondants. Les autres problématiques (rupture d'une relation, dévoilement de l'orientation sexuelle, discrimination en raison de la séropositivité, violence générale ou dans le couple, discrimination ou attitudes négatives envers l'identité de genre), ne sont rapportées que par une minorité de répondants, entre $3,2 \%$ et $12,5 \%$. Aucune différence significative n'apparaît entre les deux groupes d'âge, sauf pour la rupture d'une relation plus fréquemment rapportée chez les 55-64 ans. Toutefois, cette différence ne se maintient pas lorsque I'on tient compte des facteurs sociodémographiques (Tableau 4). 


\section{Tableau 4 Ratios de cote ajustés pour les variables de contrôle (régression logistique) comparant le groupe des 65 ans et plus au groupe des 55-64 ans}

65 ans et plus (comparativement aux 55-64 ans)

\begin{tabular}{llc} 
& RC (IC 95 \%) & $\boldsymbol{p}$ \\
\hline Auto-évaluation de la santé mentale & $2,23(1,16-4,27)$ & 0,016 \\
\hline Difficultés de santé mentale & $0,37(0,15-0,91)$ & 0,031 \\
\hline PVVIH & $0,29(0,10-0,84)$ & 0,022 \\
\hline Image corporelle & $0,60(0,33-1,09)$ & 0,091 \\
\hline Solitude ou isolement & $0,49(0,24-1,02)$ & 0,057 \\
\hline Dépression & $0,38(0,18-0,80)$ & 0,011 \\
\hline Troubles de I'alimentation & $0,34(0,07-1,61)$ & 0,173 \\
\hline Sexualité compulsive & $0,39(0,15-1,02)$ & 0,054 \\
\hline Rupture d'une relation & $0,44(0,14-1,39)$ & 0,163 \\
\hline
\end{tabular}

† Pour toutes les variables, les RC sont ajustés pour la langue du questionnaire (français ou anglais), I'orientation sexuelle, le statut relationnel, le statut d'emploi, le revenu, le niveau de scolarité, la pratique religieuse. Tous les ratios de cote ont aussi été ajustés pour l'infection par le VIH, sauf pour le modèle qui fait du statut sérologique la variable dépendante.

\section{DISCUSSION}

Les résultats de cette enquête indiquent que les deux groupes d'âge ne présentent pas de différences très marquées quant aux caractéristiques de santé et aux préoccupations relatives à la santé et aux relations interpersonnelles.

\section{Profils de santé}

L'évaluation subjective de l'état de santé générale rejoint celle recueillie dans la population canadienne générale (Statistique Canada, 2010a). Selon ces statistiques, parmi les 45-64 ans, 56,9\% des répondants 
masculins considéraient leur santé comme très bonne ou excellente, ce qui se rapproche du pourcentage rapporté parmi nos répondants dans le groupe d'âge des 55-64 ans (58,1\%). Par contre, les répondants gais et bisexuels âgés de 65 ans et plus sont plus nombreux à évaluer leur santé comme très bonne ou excellente que dans la population en général $(65,8 \%$ c. $39,3 \%)$.

Sur le plan de la santé mentale, les résultats sont aussi semblables. Dans la population générale, 73, $6 \%$ des hommes âgés de 45 à 64 ans considèrent leur santé mentale comme très bonne ou excellente, ce qui rejoint le pourcentage rapporté chez les 55-64 ans, 70,2\% dans notre échantillon. Par contre, cette évaluation positive est plus répandue parmi les 65 ans et plus $(82,8 \%)$ que dans la population en général (70,1\%; Statistique Canada, 2010b). L'avancée en âge ne semble donc pas s'accompagner de la perception d'une détérioration notable de la santé, en particulier chez les plus âgés. Quant au pourcentage de personnes vivant avec le VIH (11,6\%), il concorde avec celui rapporté dans les statistiques canadiennes de 2008 chez les 50 ans et plus estimé à 12,4\% (Agence de santé publique du Canada, 2010).

Le pourcentage des personnes souffrant d'au moins une maladie chronique est comparable à celui rapporté dans la population canadienne des 45 à 64 ans (hommes et femmes inclus) qui ont rapporté l'une ou l'autre des 11 affections chroniques dominantes. Il est de $48 \%$ dans la population canadienne (ICIS, 2011) et de 49,7\% chez les 55-64 ans. Par contre, ce pourcentage ( $74 \%$ ) est plus élevé chez les 65 ans et plus dans la population canadienne que chez nos répondants de ce même groupe d'âge $(50,0 \%)$.

Pour la santé mentale, les pourcentages rapportés par notre souséchantillon sont plus élevés que dans la population générale du même âge en 2003. Selon l'enquête de Statistique Canada (2003), 8\% des personnes dans le groupe d'âge des 45-64 ans rapportaient des symptômes reliés à I'un des troubles mentaux ou à une dépendance à des drogues, alors que ce pourcentage, sans tenir compte de l'usage des drogues, atteignait le double dans notre sous-échantillon (16,8\%). Ce pourcentage était de $3 \%$ chez les 65 ans et plus de la population générale, soit trois fois moins que dans notre population $(9,1 \%)$. La santé mentale constitue une préoccupation significative pour les hommes gais et bisexuels de 55 ans et plus, confirmant les résultats d'autres études sur les mêmes groupes (Brennan et al., 2010). 
Le cinquième des répondants rapportait un problème de santé sexuelle (principalement le VIH, I'herpès et la dysfonction érectile), ce qui semble indiquer une plus faible occurrence que dans un échantillon de population canadienne âgée ayant consulté un médecin pour un problème sexuel (Grover et al., 2006). Selon cette étude, parmi les 50-59 ans, le pourcentage de ceux qui rapportaient une dysfonction érectile modérée ou sévère était de près de $30 \%$, de près de $50 \%$ chez les $60-69$ ans et de $80 \%$ chez les 70 ans et plus. Les problèmes sexuels peuvent être aussi associés aux maladies chroniques qui affectent la libido (diabète, maladies cardiaques, médicaments, etc. ; [Wierzalis et al., 2006]) ou constituer des effets secondaires des médicaments visant à soigner des conditions médicales dont la fréquence augmente avec l'âge.

\section{Préoccupations de santé}

Pour les préoccupations majeures dans les différentes sphères de vie, peu de différences apparaissent entre les groupes d'âge. C'est le cas de la santé physique où les préoccupations à l'égard du cancer et des problèmes cardiaques viennent interpeller respectivement $20,5 \%$ et $16,4 \%$ des répondants, ce qui pourrait correspondre aux pourcentages des personnes souffrant de ces affections dans l'échantillon. Quant aux préoccupations touchant la santé mentale, la question de l'image corporelle est dominante, en particulier chez les 55-64 ans (42,3\%). Cette problématique peut être rattachée à celle des troubles de l'alimentation qui affectent aussi significativement ce groupe d'âge. Ces données rejoignent les résultats de recherches qui rapportent une plus grande prévalence de troubles de l'alimentation parmi les hommes gais et bisexuels, comparativement aux hommes hétérosexuels (Feldman et Meyer, 2007). L'importance des idéaux de minceur et de musculature dans l'expression de la masculinité et la séduction, pour cette population, serait amplifiée par les représentations véhiculées par les médias gais (Kane, 2010) et ils pourraient jouer un rôle dans les préoccupations relatives à l'image corporelle et aux troubles de la conduite alimentaire. Ces problématiques se résorbent dans le cas des personnes appartenant au groupe plus âgé qui peuvent s'être habituées au vieillissement corporel et l'avoir mieux accepté.

Viennent ensuite les questions entourant l'anxiété (35,4\%), la solitude et l'isolement $(27,9 \%)$ et la dépression $(26,1 \%)$ qui préoccupent plus 
le groupe des 55-64 ans. La première fourchette d'âge semble donc constituer une période critique dans le champ de la santé mentale, même si ces préoccupations se réduisent quelque peu par la suite. Ces résultats rejoignent ceux d'autres études qui montrent que les problèmes d'anxiété et de dépression sont plus fréquents chez les hommes gais et bisexuels (King et al. 2008; Mayock et al. 2009; Brennan et al., 2010 ; Johnston, 2008). Les préoccupations relatives aux idéations et aux tentatives suicidaires rejoignent aussi près de $10 \%$ des répondants. Ce pourcentage est plus important que celui relevé dans la population âgée canadienne ( $2 \%$; Corna et al., 2010), mais il confirme les tendances rapportées dans la population des gais et des bisexuels (Brennan et al., 2010).

La dépendance aux images érotiques et à Internet constitue un problème pour $20 \%$ des répondants, sans différence entre les groupes d'âge. Cette préoccupation suggère que tout comme pour les personnes âgées dans la population en général, Internet constitue un outil permettant d'accéder facilement à des contenus sexuels qui peuvent alimenter les fantasmes et les pratiques sans exposer à une stigmatisation ou à des répercussions négatives (Parsons, Severino, Grov, Bimbi et Morgenstern, 2007). Toutefois, un usage abusif peut conduire à une dépendance lorsqu'elles ne peuvent en faire une gestion adéquate (Parsons et al., 2007), une assuétude qui se reflète aussi dans le champ de la compulsion sexuelle qui rejoint $17 \%$ des répondants, en particulier les 55-64 ans, plus enclins à relever ce problème que les 65 ans et plus. Ce type de difficultés de santé ne semble pas avoir fait l'objet de recherches empiriques approfondies, ce qui empêche une compréhension adéquate tant parmi les minorités sexuelles que chez les hétérosexuels en processus de vieillissement. Par contre, la dépendance au jeu ne touche qu'une faible minorité de répondants $(2 \%)$, un pourcentage qui rejoint celui décelé par les recherches épidémiologiques touchant le jeu pathologique dans la population adulte canadienne, tous âges confondus pour une période 12 mois (Cox et al., 2005).

L'acceptation de l'orientation sexuelle de même que la crainte qu'elle ne soit découverte continuent d'être des questions préoccupantes pour près de $20 \%$ des répondants. Elles peuvent être liées en partie à la peur de la discrimination ou à des attitudes négatives de l'entourage à l'égard des orientations sexuelles non-exclusivement hétérosexuelles, 
rapportées aussi par près de $18 \%$ des répondants. Comme le montre I'Enquête sociale générale canadienne de 2004 (Beauchamp, 2008), une majorité de personnes gaies et bisexuelles qui avait vécu des formes de discrimination l'attribuait à leur orientation sexuelle et ces configurations rejoignent celles rapportées parmi les gais et les bisexuels irlandais (Higgins et al.,2011). L'acceptation de l'identité de genre pose aussi problème pour environ $10 \%$ des répondants, tout comme celle du dévoilement de son orientation. Ces données, dans leur ensemble, suggèrent que pour un pourcentage significatif de répondants, I'orientation sexuelle continue de constituer une dimension problématique, sans doute en raison des attitudes homophobes encore répandues au Canada qui font craindre la discrimination.

Dans le champ de la santé sexuelle, le tiers des répondants rapportent des préoccupations associées au dépistage du VIH/sida et aux ITSS, ainsi qu'aux conduites sexuelles à risque, sans différence entre les deux groupes d'âge, alors que $20 \%$ sont préoccupés par la prévention du VIH. Ces tendances suggèrent que malgré I'avancée en âge, une proportion significative de gais et bisexuels continue d'être attentive à des problèmes qui affectent de façon importante leurs communautés et auxquels ils continuent d'être exposés (Anderson, 2009; Wierzalis et al., 2006).

Peu de recherches se sont attachées à dégager les patrons de consommation de drogues, d'alcool et de tabac parmi les hommes gais et bisexuels âgés (Satre, 2006; Anderson, 2009), mais parmi les LGBT âgés dans leur ensemble, leur prévalence serait plus élevée que dans la population hétérosexuelle âgée. Au Canada, l'étude de Brennan et al. (2010) indique que le pourcentage des fumeurs quotidiens est de $26,2 \%$ chez les homosexuels et de $27,2 \%$ chez les bisexuels, des chiffres un peu plus élevés que dans la population hétérosexuelle $(21,1 \%)$. Dans le groupe masculin canadien des $45-54$ ans, il est de $22,8 \%$ et chez les 55 ans et plus, de 12,3\% (Santé Canada, 2011). Dans notre échantillon, le tabac est la préoccupation la plus importante parmi les habitudes de consommation et elle interpelle le quart des répondants, sans différence entre les groupes d'âge. Quant à la consommation de drogues, parmi les 45-54 ans, les données canadiennes montrent que $11 \%$ avaient consommé du Cannabis dans les 12 derniers mois avant l'enquête, mais ce pourcentage diminuait à $5,6 \%$ chez les 55-64 ans, les données étant manquantes pour les 
groupes d'âge supérieurs (Santé Canada, 2008). Seul un faible pourcentage d'hommes gais et bisexuels de notre enquête se disait préoccupé par sa consommation de drogues $(2,0 \%)$, ce chiffre suggérant qu'un pourcentage plus important en consomme.

Selon l'enquête de Brennan et al. (2010), le pourcentage des consommateurs d'alcool à risque est respectivement de $11,1 \%$ chez les homosexuels, $16,3 \%$ chez les bisexuels et $13,3 \%$ chez les hétérosexuels. Les statistiques canadiennes sur les personnes âgées montrent que parmi les hommes de 45-64 ans, 15,5\% avaient eu une consommation abusive d'alcool en 2010 et ce pourcentage descendait à 7,7\% chez les 64 ans et plus (Statistiques Canada, 2011). Dans la présente enquête, la proportion des répondants préoccupés par leur consommation apparaît relativement similaire (en moyenne 13,5\%). Ces configurations suggèrent que dans notre échantillon les hommes gais et bisexuels sont confrontés à des soucis quant à l'usage du tabac et de l'alcool qui rejoignent les tendances comportementales rapportées dans les populations âgées du Canada.

Dans la sphère des relations interpersonnelles, les préoccupations associées à la quête d'un partenaire stable ou d'amis sont rapportées par un pourcentage important de répondants (respectivement, 28,6\% et $25,1 \%$ ), ce qui reflète les enjeux entourant la solitude et l'isolement vécus par les personnes âgées, plus de la moitié de nos répondants ne rapportant pas de relations de couple et $12,5 \%$ étant préoccupés par une rupture dans le couple. Ce pourcentage est plus élevé que dans la population canadienne générale, se situant autour de $20 \%$ pour le groupe des 50-64 ans, selon les données du recensement de 2006 et se maintenant chez les 64 ans et plus, sauf chez les personnes âgées de 80 ans et plus (Statistiques Canada, 2011). Le cinquième des répondants exprime des préoccupations face à la relation de couple actuelle et aux relations familiales. Les autres préoccupations (violence générale ou dans le couple, discrimination ou attitudes négatives envers I'identité de genre) sont rapportées par une minorité de répondants (entre $8,6 \%$ et $3,2 \%$ ) et occupent donc une place très secondaire dans les problématiques de santé. 


\section{CONCLUSIONS}

Malgré les limites méthodologiques de notre recherche liées au recrutement en ligne et donc à la représentativité de notre sous-échantillon, aux effectifs réduits du groupe d'âge des 65 ans et plus ainsi qu'à l'usage d'une liste de préoccupations dont le contenu précis reste à déterminer, cette étude met en évidence l'importance de certains problèmes de santé déjà soulignés dans les études sur le vieillissement parmi les minorités sexuelles. Dans le domaine physique on constate la présence de maladies chroniques, mais surtout la persistance de certains enjeux propres aux hommes gais et bisexuels, comme la prévention et le dépistage du VIH, des ITSS et les comportements sexuels à risque. C'est surtout dans le champ de la santé mentale que les problèmes semblent plus significatifs. Les questions de l'image corporelle, de la dépression et de l'anxiété, l'acceptation de l'orientation sexuelle, mais aussi celles relatives à la solitude et à l'isolement ou à la difficulté à trouver un partenaire stable occupent une place importante. La comparaison de sous-groupes d'âge en fonction des variables de contrôle indiquent que les écarts entre les 55-64 ans et les 65 ans sont faibles, bien que le premier groupe semble à plusieurs égards plus préoccupé que le groupe plus âgé. Ces résultats suggèrent l'importance de soutenir les hommes gais et bisexuels pour les aider à se préparer à bien vieillir et que ce soutien doit être maximal auprès des 55-64 ans. D'autres recherches sont cependant nécessaires pour mieux comprendre la spécificité des profils et des préoccupations de santé dans cette population mais aussi leurs modes de résilience.

\section{BIBLIOGRAPHIE}

AGENCE DE LA SANTÉ PUBLIQUE DU CANADA (2010). Chapitre 6. L'infection à VIH et le sida chez les personnes âgées au Canada. <http://www. phac-aspc.gc.ca/aids-sida/publication/epi/2010/6-fra.php>, consulté le 13 février 2012.

ANDERSON, S. (2009). Substance use disorders in lesbian, gay, bisexual and transgender clients, New York, Columbia University Press.

BEAUCHAMP, D. L. (2008). Sexual orientation and victimization, Ottawa, Canadian Centre for Justice Statistics, Statistics Canada.

BROTMAN, S., B. RYAN et R. CORMIER (2003). «The health and social service needs of gay and lesbian elders and their families in Canada», The Gerontologist, vol. 43, n², p. 192-202.

BRENNAN, D. J. et al. (2010). «Men's sexual orientation and health in Canada», Canadian Journal of Public Health, vol. 101, n 3, p. 255-258. 
Committee on Lesbian, Gay, Bisexual, and Transgender Health Issues and Research Gaps and Opportunities Board on the Health of Select Populations (2011). Health of Lesbian, Gay, Bisexual and Transgender (LGBT) People: Building a Foundation for Better Understanding, Washington, National Academies Press.

CORNA, L. et al. (2010). «Suicide ideation in older adults: relationship to mental health problems and service use», The Gerontologist, vol. 50, n० 6, p. $785-797$.

COX, B.J., N. YU, T. AFIFI et R. LADOUCEUR (2005). «A national survey of gambling problems in Canada», Canadian Journal of Psychiatry, vol. 50, n० 4 p. 213-217.

DYSART-GALE, D. (2010). «Social justice and social determinants of health: lesbian, gay, bisexual, transgendered, intersexed, and queer youth in Canada », Journal of Child and Adolescent Psychiatric Nursing, vol. 23, $\mathrm{n}^{\circ} 1$, p. 23-28.

FELDMAN, M. B. et I.H. MEYER (2007). «Eating disorders in diverse lesbian, gay, and bisexual populations», International Journal of Eating Disorders, vol. 40, p. 218-226.

FREDERIKSEN-GOLDSEN, K.I. et A. MURACO (2010). «Aging and sexual orientation: 25 years review of the literature», Research on Aging, vol. 32, $\mathrm{n}^{\circ} 3$, p. 372-413.

GRANT, J.M. (2010). Outing Age 2010. Public policy issues affecting lesbian, gay, bisexual and transgender elders, New York, National Gay and Lesbian Task Force.

GROVER, S.A. et al. (2006). «The prevalence of erectile dysfunction in the primary care setting: importance of risk factors for diabetes and vascular disease », Archives of Internal.Medicine, vol. 166, n² 2, p. 213-219.

HAAS, A. P., M. ELIASON, V. M. MAYS, R. M. MATHY et al. (2011). «Suicide and suicide risk in lesbian, gay, bisexual, and transgender populations: Review and recommendations», Journal of Homosexuality, vol. 58, p. 10-51.

HIGGINS, A. et al. (2011). Visible Lives. Identifying the experiences and needs of older Lesbian, Gay, Bi-sexual and Transgender people in Ireland, Gay and Lesbian equality Network (GLEN), Dublin, Irlande.

JOHNSTON, A. (2008). Exploring the health needs of older lesbian and gay men in metro Vancouver. Thèse de maîtrise, Département de Gérontologie, Simon Frazer University, Vancouver.

ICIS (2011). Les personnes âgées et le système de santé: quelles sont les répercussions des multiples affections chroniques? <Air-chronic_disease_ aib_fr-2.pdf>.

KING, M. et al. (2008). «A systematic review of mental disorder, suicide and deliberate self harm in lesbian, gay and bisexual people», BMC Psychiatry, vol. $8, n^{\circ} 70$. doi : $10.1186 / 1471-244 X-8 / 70$

MAYOCK, P. et al. (2009). Supporting LGBT lives: A study of the mental health and well-being of Lesbian, gay, bisexual and transgender people, Gay and Lesbian equality Network (GLEN) and BeLonG To Youth service, Dublin, Irlande.

MEYER, I. H. et M.E. NORTHBRIDGE (dir.), (2007). The health of sexual minorities: Public health perspectives on lesbian, gay, bisexual and transgender populations, New York, Springer. 
MEYER, I. H. (2007). «Prejudice and discrimination as social stressors», dans I. H. Meyer et M. E. Northbridge (dir.), The health of sexual minorities: Public health perspectives on lesbian, gay, bisexual and transgender populations, New York, Springer, p. 242-267.

PARSONS, J. T. et al. (2007). «Internet use among gay and bisexual men with compulsive sexual behavior», Sexual Addiction \& Compulsivity, vol. 14, p. 239-256.

SAEWYC, E. M. et al. (2009). «Protective factors in the lives of bisexual adolescents in North America», American Journal of Public Health, vol. 99, $\mathrm{n}^{\circ} 1$, p. 110-117.

SANTÉ CANADA (2008). Enquête sur les toxicomanies au Canada (ETC). <http://www.hc-sc.gc.ca/hc-ps/pubs/adp-apd/cas_gender-etc_sexe/ chap4_tbl1-fra.php\#tbl4-2>, consulté le 13 février 2012.

SATRE, D.D. (2006). «Use and misuse of alcohol and drugs», dans D. KIMMEL et al. (dir.), Lesbian, gay, bisexual and transgender aging, New York, Columbia University Press, p. 131-151.

SMITH, M. (2005). «Explaining human rights protections: Institutionalist analysis in the lesbian and gay rights case», Communication présentée à I'Annual Meeting of the Canadian Political Science Association, University of Western Ontario, London, Ontario, <http://www.cpsa-acsp.ca/papers-2005/ Smith,\%20Miriam.pdf>.

STALL, R. D, M. FREEMAN et J.A. CATANIA (2008). «Interacting epidemics and gay men's health: A theory of syndemic production among urban gay men», dans R. J. Wolitski et al. (dir.), Unequal opportunity: Health disparities affecting gay and bisexual men in the United States, New York, Oxford University Press, p. 251-274.

STATISTICS CANADA (2011). Population in couples by age group and sex, Canada, 2006, <http://www.statcan.gc.ca/pub/89-503-x/2010001/article/ 11546/tbl/tbl002-eng.htm>, consulté le 15 février 2012.

STATISTIQUE CANADA (2003). Canadian Community Health Survey: Mental health and well-being, <http://www.statcan.gc.ca/daily-quotidien/030903/ dq030903a-eng.htm>, consulté le 13 février 2012.

STATISTIQUE CANADA (2010a). Santé perçue selon le groupe d'âge et le sexe, <http://www.statcan.gc.ca/pub/82-229-x/2009001/status/phx-fra.htm>, consulté le 13 février 2012.

STATISTIQUE CANADA (2010b). Santé mentale perçue selon le groupe d'âge et le sexe, <http://www.statcan.gc.ca/pub/82-229-x/2009001/status/pmh-fra. htm>, consulté le 13 février 2012.

STATISTIQUE CANADA (2011). Consommation abusive d'alcool, selon le groupe d'âge et le sexe, <http://www40.statcan.ca/I02/cst01/health79bfra.htm>, consulté le 13 février 2012.

TIEPKEMA, M. (2008). «Health care use among gay, lesbian and bisexual Canadians», Health Reports, vol. 19, n० 1, p. 53-64.

WIERZALIS, E.A., B. BARRET, M. POPE et M. RANKINS (2006). «Gay men and aging: sex and intimacy», dans D. KIMMEL et al. (dir.), Lesbian, gay, bisexual and transgender aging, New York, Columbia University Press, p. 91-109. 


\section{NOTES}

1. Cette recherche a été rendue possible grâce à une subvention des Instituts de recherche en santé du Canada (RSC 2009-03-MOP-201493) et celle du Fonds québécois de recherche sur la société et la culture (FQRSC) attribuée à l'équipe de recherche SVR (Sexualités, Vulnérabilités et Résilience) de I'UQAM.

2. Selon la Fédération canadienne pour la santé sexuelle, «le terme «bispirituel-le» (ou «à deux esprits») désigne des personnes qui ne sont considérées ni comme des hommes ni comme des femmes. Ceci implique souvent un esprit masculin et un esprit féminin qui habitent le même corps.», <http://www.cfsh.ca/fr/your_sexual_health/gender-identityand-sexual-orientation/transgendered-and-twospirited.aspx>, consulté le 19 février 2012. 